\title{
SYNTHESIS AND MICROBIOLOGICAL EVALUATION OF MANNICH BASES DERIVED FROM 4,6-DIACETYLRESORCINOL
}

\author{
ASIF HUSAIN ${ }^{*}$, MOHAMMAD MAAZ², KHURSHEED AHMAD ANSARI ${ }^{2}$ AUSAF AHMAD ${ }^{3}$, MOHD. RASHID \\ ${ }^{1}$ Department of Pharmaceutical Chemistry, Faculty of Pharmacy, Jamia Hamdard (Hamdard University), New Delhi- 110062 , India; ${ }^{2}$ Faculty of Medicine \\ (Unani), Jamia Hamdard (Hamdard University), New Delhi-110 062, ${ }^{3}$ Department of Biochemistry and Biophysics, \\ University of Rochester Medical Center, Rochester, NY 14642, USA \\ (Received: September 14, 2009 - Accepted: June 15, 2010)
}

\section{SUMMARY}

In the present investigations 4,6-diacetyl resorcinol 1 on condensation with formaldehyde and some selected amines following the Mannich reaction conditions yielded eight new Mannich bases 2a-h. It was observed that the reaction did not take place at the acetyl function but occurred at the 2-position into the aromatic ring. The compounds were characterized on the basis of elemental analysis as well as ${ }^{1} \mathrm{H}$ NMR and Mass spectral data. The antibacterial and antifungal activities of the title compounds were tested by the disc diffusion method using nutrient agar medium against various microorganisms such as gram positive Staphylococcus aureus and Bacillus subtilis, gram negative Escherichia coli and the fungi Aspergillus flavus and Candida albicans. Ofloxacin and voriconazole at $20 \mu \mathrm{g} / \mathrm{mL}$ were used as standard drugs for antibacterial and antifungal activities, respectively.

Keywords : Mannich base, resorcinol, antibacterial, antifungal.

\section{INTRODUCTION}

The presence of nitrogen atom alongwith other features impart interesting biological activities to the parent compounds' ${ }^{1}$. Mannich bases, including those derived from different acetophenones, possess diverse biological activities including potent antibacterial ${ }^{2,4}$, antimycobacterial ${ }^{3}$, antifungal ${ }^{2,4}$ and anti-HIV ${ }^{4}$ activities. Resorcinol is a simple and important aromatic chemical (1,3-benzenediol) that has been chemically incorporated into various compounds to enhance their pharmacological profile ${ }^{5}$.

The essential feature of Mannich reaction is the replacement of the active hydrogen by an aminomethyl or substituted aminomethyl group. However, it is also well known that with phenols the reaction proceeds on a nuclear position. Hence it was considered worthwhile to study this reaction on 4,6-diacetyl resorcinol having both $\mathrm{COCH}_{3}$ and nuclear position available. This moiety was used earlier to build benzodipyrone derivatives and found to have a good antibacterial activity ${ }^{6}$.

Over the past few decades the bacterial resistance to antibiotics has become one of the most important problems of infections treatment. Although there are antimicrobial agents having different structures and mechanisms are frequently used in the treatment of microbial infections, even then these agents are associated with resistance. To overcome the development of drug resistance, it is necessary to synthesize a new class of antimicrobial compounds possessing different mechanism or chemical properties from those that are used commonly. Derivatives of 4,6-diacetyl resorcinol show potential antibacterial activity ${ }^{6}$. In view of these points and in continuation of our work on novel resorcinol derivatives ${ }^{6}$, it was planned to synthesize Mannich bases derived from 4,6-diacetyl resorcinol.

In the present work we report the Mannich reaction proceeds at the 2-position of 4,6-diacetyl resorcinol and several new such compounds have been prepared by varying amine component and the products have been evaluated for their antibacterial and antifungal activities.

\section{EXPERIMENTAL}

\section{Chemistry}

Melting points were determined in open capillary tubes and are uncorrected. Purity of the compounds was checked by thin layer chromatography (TLC) on silica gel G plates, with the solvent system: toluene-ethyl acetate-formic acid $(5: 4: 1, v / v / v)$. The spots were located under iodine vapours and UV light. ${ }^{1} \mathrm{H}$ NMR spectra were recorded on a Bruker $300 \mathrm{MHz}$ NMR spectrometer (internal reference-tetramethyl silane) and Mass spectra were recorded on a JEOL JMS-D 300 instrument. Elemental analyses were performed on a PerkinElmer 240 analyzer and the values were in range of $\pm 0.4 \%$ for each element analyzed $(\mathrm{C}, \mathrm{H}, \mathrm{N})$.

Preparation of 1,1'-(4,6-dimethyl-1,3-phenylene)diethanone (1)
It was synthesized according to the method reported in literature ${ }^{6}$.

Preparation of Mannich bases (2a-h)

Compound $\mathbf{1}(0.002 \mathrm{~mol})$ was dissolved in chloroform $(20 \mathrm{ml})$ and to it were added formaldehyde $(0.004 \mathrm{~mol})$, an amine $(0.004 \mathrm{~mol})$ and tetrabutyl ammonium bromide $(0.002 \mathrm{~mol})$ followed by distilled water $(10 \mathrm{ml})$. After stirring the contents for $24 \mathrm{~h}$, chloroform layer was separated and washed with aqueous sodium bicarbonate $(5 \% \mathrm{w} / \mathrm{v})$ followed by washing with water. The organic layer was then dried over anhydrous sodium sulphate, filtered and evaporated to dryness. The residue was crystallized from an appropriate solvent to give TLC pure crystals of 2a-h (Table I). Each one of these compounds gave a violet colour with ethanolic ferric chloride solution (test for phenol).

1,1'-\{5-[(Dimethylamino)methyl]-4,6-dihydroxy-1,3-phenylene $\}$ diethanone (2a): Yield: $63 \%$; m.p. $148-150{ }^{\circ} \mathrm{C} ;{ }^{1} \mathrm{H}-\mathrm{NMR}$ (DMSO- $d_{6}, \delta$, ppm): $1.32\left(\mathrm{~s}, 6 \mathrm{H}, 2 \mathrm{x}-\mathrm{CH}_{3}\right), 2.61$ (s, $\left.6 \mathrm{H}, 2 \mathrm{x}-\mathrm{COCH}_{3}\right), 4.13\left(\mathrm{~s}, 2 \mathrm{H},-\mathrm{CH}_{2}-\right.$, benzylic), 8.27 (s, 1H, aromatic proton), $13.18(\mathrm{~s}, 2 \mathrm{H}, 2 \mathrm{xOH}) ; \mathrm{MS}(\mathrm{m} / \mathrm{z}): 251\left(\mathrm{M}^{+}\right), 207$, 193, 190, 44; Anal. $\mathrm{C}_{13} \mathrm{H}_{17} \mathrm{NO}_{4}$, Calcd. C, 62.14; H, 6.82; N, 5.57; found C, $62.18 ; \mathrm{H}, 6.86 ; \mathrm{N}, 5.45$.

1,1'-\{5-[(Diethylamino)methyl]-4,6-dihydroxy-1,3-phenylene\}diethanone (2b): Yield: $60 \%$; m.p. 135-137 ${ }^{\circ} \mathrm{C}$; ${ }^{1} \mathrm{H}-\mathrm{NMR}$ (DMSO- $d_{6}, \delta$, ppm): 1.35 (t, $\left.6 \mathrm{H}, 2 \mathrm{x}-\mathrm{CH}_{2} \mathrm{CH}_{3}\right), 2.87$ (q, 4H, 2x- $\left.\mathrm{CH}_{2} \mathrm{CH}_{3}\right), 2.60\left(\mathrm{~s}, 6 \mathrm{H}, 2 \mathrm{x}-\mathrm{COCH}_{3}\right), 4.08$ (s, $2 \mathrm{H},-\mathrm{CH}_{2}-$, benzylic), 8.35 (s, $1 \mathrm{H}$, aromatic proton), 13.25 (s, $\left.2 \mathrm{H}, 2 \mathrm{xOH}\right) . \mathrm{MS}$ $(\mathrm{m} / \mathrm{z}): 279\left(\mathrm{M}^{+}\right), 207,193,190,72$. Anal. $\mathrm{C}_{15} \mathrm{H}_{21} \mathrm{NO}_{4}$, calcd. C, 64.50; H, 7.58; $\mathrm{N}, 5.01$; found $\mathrm{C}, 64.62 ; \mathrm{H}, 7.54 ; \mathrm{N}, 5.07$.

$1,1^{\prime}-\{5-[(4-M e t h y l p h e n y l a m i n o) m e t h y l]-4,6-d i h y d r o x y-1,3-p h e n y l e n e\}$ diethanone (2c): Yield: $52 \%$; m.p. $167-169{ }^{\circ} \mathrm{C}$; ${ }^{1} \mathrm{H}-\mathrm{NMR}$ (DMSO- $d_{6}, \delta$, ppm): $1.35\left(\mathrm{~s}, 3 \mathrm{H},-\mathrm{CH}_{3}\right), 2.67\left(\mathrm{~s}, 6 \mathrm{H}, 2 \mathrm{x}-\mathrm{COCH}_{3}\right), 4.14\left(\mathrm{~s}, 2 \mathrm{H},-\mathrm{CH}_{2}-\right.$, benzylic), $7.03 \& 7.46\left(\mathrm{~d}\right.$, each, $\mathrm{A}_{2} \mathrm{~B}_{2}, p$-substituted phenyl ring), 8.37 (s, $1 \mathrm{H}$, aromatic proton), 9.02 (s, 1H, -NH-), $13.18(\mathrm{~s}, 2 \mathrm{H}, 2 \mathrm{xOH}) ; \mathrm{MS}(\mathrm{m} / \mathrm{z}): 313\left(\mathrm{M}^{+}\right), 207$, 91, 77; Anal. $\mathrm{C}_{18} \mathrm{H}_{19} \mathrm{NO}_{4}$, calcd. C, 69.00; H, 6.11; N, 4.47; found C, 68.88; H, $6.20 ; \mathrm{N}, 4.48$.

1,1'-\{5-[(4-Methoxylphenylamino)methyl]-4,6-dihydroxy-1,3-phenylene\} diethanone (2d): Yield: $58 \%$; m.p. $172-174{ }^{\circ} \mathrm{C}$; ${ }^{1} \mathrm{H}-\mathrm{NMR}$ (DMSO- $d_{6}, \delta$, ppm): $2.73\left(\mathrm{~s}, 6 \mathrm{H}, 2 \mathrm{x}-\mathrm{COCH}_{3}\right), 3.91\left(\mathrm{~s}, 3 \mathrm{H},-\mathrm{OCH}_{3}\right), 4.18\left(\mathrm{~s}, 2 \mathrm{H},-\mathrm{CH}_{2}-\right.$, benzylic), $7.14 \& 7.57$ (d, each, $\mathrm{A}_{2} \mathrm{~B}_{2}, p$-substituted phenyl ring), 8.52 (s, $1 \mathrm{H}$, aromatic proton), 9.06 (s, $1 \mathrm{H},-\mathrm{NH}-), 13.22(\mathrm{~s}, 2 \mathrm{H}, 2 \mathrm{xOH}) ; \mathrm{MS}(\mathrm{m} / \mathrm{z}): 329\left(\mathrm{M}^{+}\right), 207$, 193, 190, 122; Anal. $\mathrm{C}_{18} \mathrm{H}_{19} \mathrm{NO}_{5}$, calcd. C, 65.64; H, 5.81; N, 4.25; found C, 65.57; H, 5.76; N, 4.31 .

1,1'-\{5-[(3-Methoxylphenylamino)methyl]-4,6-dihydroxy-1,3-phenylene\} diethanone (2e): Yield: $55 \%$; m.p. $160-162^{\circ} \mathrm{C}$; ${ }^{1} \mathrm{H}-\mathrm{NMR}$ (DMSO- $d_{6}, \delta$, ppm): $2.69\left(\mathrm{~s}, 6 \mathrm{H}, 2 \mathrm{x}-\mathrm{COCH}_{3}\right), 3.88\left(\mathrm{~s}, 3 \mathrm{H},-\mathrm{OCH}_{3}\right), 4.16\left(\mathrm{~s}, 2 \mathrm{H},-\mathrm{CH}_{-}-\right.$, benzylic $)$, 7.08-7.33 (m, 4H, $m$-substituted phenyl ring), 8.29 (s, $1 \mathrm{H}$, aromatic proton), 8.97 (s, 1H, -NH-), 13.17 (s, 2H, 2xOH); MS (m/z): $329\left(\mathrm{M}^{+}\right), 207,193,190$, 122, 121; Anal. $\mathrm{C}_{18} \mathrm{H}_{19} \mathrm{NO}_{5}$, calcd. C, 65.64; H, 5.81; N, 4.25; found C, 65.66; $\mathrm{H}, 5.78 ; \mathrm{N}, 4.22$.

1,1'-\{5-[(Morpholin-4-yl)methyl]-46-dihydroxy-1,3-phenylene $\}$ 
diethanone (2f): Yield: $64 \%$; m.p. $134-136{ }^{\circ} \mathrm{C} ;{ }^{1} \mathrm{H}-\mathrm{NMR}$ (DMSO- $d, \delta$, ppm): 2.68 (b, $10 \mathrm{H}, 2^{\prime}-\mathrm{COCH}_{3}$ and $2 \mathrm{x}-\mathrm{CH}_{2}-$, a to nitrogen), 3.87 (b, 6H, $2^{\prime}-\mathrm{CH}_{2}-$, a to oxygen + $-\mathrm{CH}_{2}$-, benzylic), 8.35 (s, $1 \mathrm{H}$, aromatic proton), $13.16(\mathrm{~s}, 2 \mathrm{H}, 2 \mathrm{xOH})$; MS (m/z): $293\left(\mathrm{M}^{+}\right), 207,193,190,86 ;$ Anal. $\mathrm{C}_{15} \mathrm{H}_{19} \mathrm{NO}_{5}$, calcd. C, 61.42; H, 6.53 ; N, 4.78; found C, 61.40; H, 6.51; N, 4.83 .

$1,1$ '-\{5-[(Piperidin-1-yl)methyl]-4,6-dihydroxy-1,3-phenylene $\}$ diethanone (2g): Yield: $66 \%$; m.p $138-140{ }^{\circ} \mathrm{C}$; ${ }^{1} \mathrm{H}-\mathrm{NMR}$ (DMSO- $d_{6}, \delta$, ppm): 1.67 (bs, $6 \mathrm{H}, 2^{\prime}-\mathrm{CH}_{2}$, meta to $\mathrm{N}+-\mathrm{CH}_{2}$, para to $\mathrm{N}$ ), 2.82 (s, $4 \mathrm{H}, 2^{\prime}-\mathrm{CH}_{2}$, ortho to $\mathrm{N}), 2.66\left(\mathrm{~s}, 6 \mathrm{H}, 2^{\prime}-\mathrm{COCH}_{3}\right), 3.97$ (s, $2 \mathrm{H},-\mathrm{CH}_{2}-$, benzylic), 8.34 (s, $1 \mathrm{H}$, aromatic proton), $13.23(\mathrm{~s}, 2 \mathrm{H}, 2 \mathrm{xOH}) ; \mathrm{MS}(\mathrm{m} / \mathrm{z}): 291\left(\mathrm{M}^{+}\right), 207,193,190$, 84. Anal. $\mathrm{C}_{16} \mathrm{H}_{21} \mathrm{NO}_{4}$, calcd. C, 65.96; H, 7.26; N, 4.81; found C, 65.92; $\mathrm{H}$, $7.25 ; \mathrm{N}, 4.86$

1,1'-\{5-[(4-Methylpiperazin-1-yl)methyl]-4,6-dihydroxy-1,3-phenylene\} diethanone (2h): Yield: $57 \%$; m.p. $162-164{ }^{\circ} \mathrm{C} ;{ }^{1} \mathrm{H}-\mathrm{NMR}$ (DMSO-d $d_{6}, \delta$, ppm): $2.33\left(\mathrm{~s}, 3 \mathrm{H},-\mathrm{CH}_{3}\right), 2.51$ (bs, 8H, 4x CH$), 2.72\left(\mathrm{~s}, 6 \mathrm{H}, 2^{\prime}-\mathrm{COCH}_{3}\right), 4.09$ (s, $2 \mathrm{H},-\mathrm{CH}_{2}$-, benzylic), 8.24 (s, $1 \mathrm{H}$, aromatic proton), $13.86(\mathrm{~s}, 2 \mathrm{H}, 2 \mathrm{xOH}) ; \mathrm{MS}$ $(\mathrm{m} / \mathrm{z}): 306\left(\mathrm{M}^{+}\right), 207,193,190,99$. Anal. $\mathrm{C}_{16} \mathrm{H}_{22} \mathrm{~N}_{2} \mathrm{O}_{4}$, calcd. C, 62.73; H, 7.24; N, 9.14; found C, 62.65; H, 7.26; N, 9.12.

\section{Antibacterial and antifungal activity}

All the synthesized compounds were screened for in vitro antibacterial and antifungal activities at the concentration of 100 and $200 \mu \mathrm{g} / \mathrm{mL}$ by cup-plate method $^{7}$ in nutrient agar (Hi-media) (antibacterial activity) and Sabouraud dextrose agar (Hi-media) (antifungal activity). The bacterial strains gram positive (Bacillus subtilis ATCC 6633 \& Staphylococcus aureus ATCC 25923), gram negative (Escherichia coli ATCC 8739) and fungal strains (Candida albicans NCIM 300 \& Aspergillus flavus NCIM 524) were used. The sterilized media was poured into sterile petridishes and allowed to solidify for 30 minutes. On the surface of the media, $0.1 \mathrm{ml}$ microbial suspension was spread and after 10 minutes, cups were made by punching into agar surface with a sterile cork borer ( $6 \mathrm{~mm}$ diameter) and scooping out the punched part of the agar. Four cups were made in each petridish and into these cups was added $0.1 \mathrm{ml}$ of the drug solution. The drug solution was allowed to diffuse for about an hour and plates were then incubated at $37 \pm 0.5^{\circ} \mathrm{C}$ for $24 \mathrm{~h}$ (antibacterial activity) and at $28{ }^{\circ} \mathrm{C}$ for $48-72 \mathrm{~h}$ (antifungal activity). A solvent control dimethylformamide (DMF) was also run to know the activity of the blank. The diameter of zone of inhibition $(\mathrm{mm})$ was measured. Ofloxacin $(20 \mu \mathrm{g} / \mathrm{mL})$ and voriconazole $(20 \mu \mathrm{g} / \mathrm{mL})$ were used as reference drugs for comparison. The results are presented in Table-II.

\section{RESULTS AND DISCUSSION}

\section{Chemistry}

Titled compounds (2a-h) were synthesized through one-pot reaction as depicted in Scheme-1. The starting material 4,6-diacetyl resorcinol 1 was
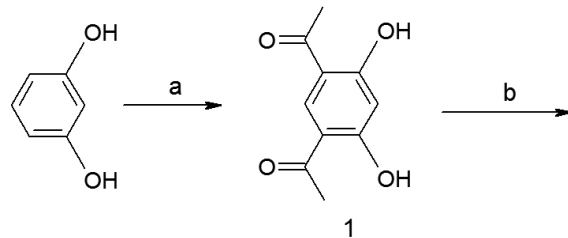

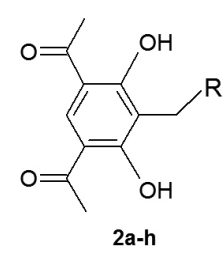

2a-h
Scheme-1. Protocol for synthesis, (a) $\mathrm{Ac}_{2} \mathrm{O} / \mathrm{ZnCl}_{2}$; (b) Tetrabutyl amm bromide/HCHO/amine

synthesized by the reported procedure ${ }^{6}$.

It (1) was condensed with different amines and formaldehyde, however, the yields were poor and hence the reaction was carried out in the presence of tetrabutyl ammonium bromide, a phase transfer catalyst, in chloroform solution to give Mannich bases $\mathbf{2 a - h}$ in satisfactory yields. The structures assigned to the compounds were supported by the results of elemental analysis as well as ${ }^{1} \mathrm{H}$ NMR and Mass spectral data (Table-I).

The formation of Mannich bases could be inferred by the presence of a methylene signal around d $4.0 \pm 0.2$ instead of the signals for $\mathrm{CH}_{2}-\mathrm{CH}_{2}$ and the presence of acetyl signal consistently around d 2.6 \pm 0.2 . Further mass spectral data of all these compounds showed molecular ion peaks of reasonable intensity besides the diagnostic peaks at m/z 207, 193 and 190 arising from diacetyl resorcinol moiety and can be formulated as shown in Fig. 1. Beside these, there was a fragment arising from the amine moiety, which was dependent on the amine used.

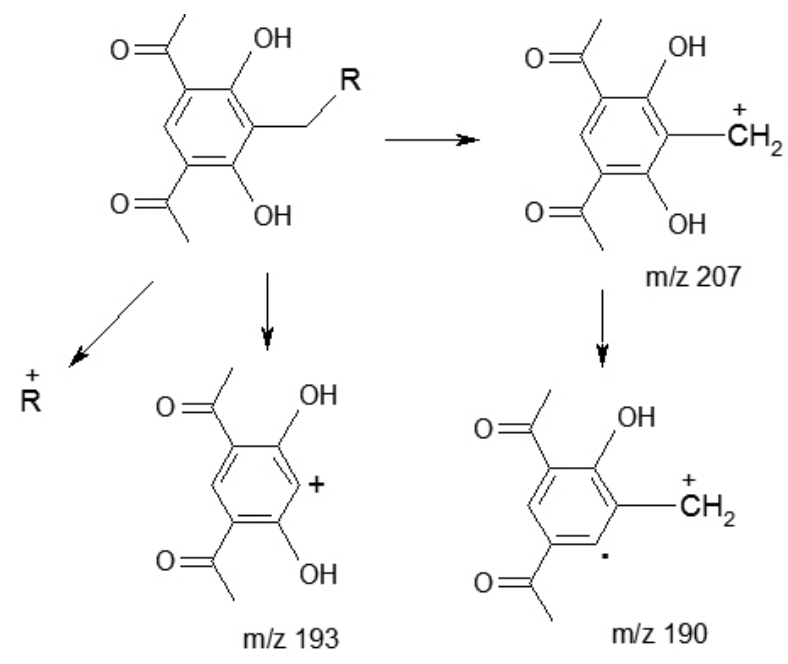

Fig.1: Mass fragmentation pattern of the compounds 2a-h.

Table-I: Physical data of the compounds 2a-h.

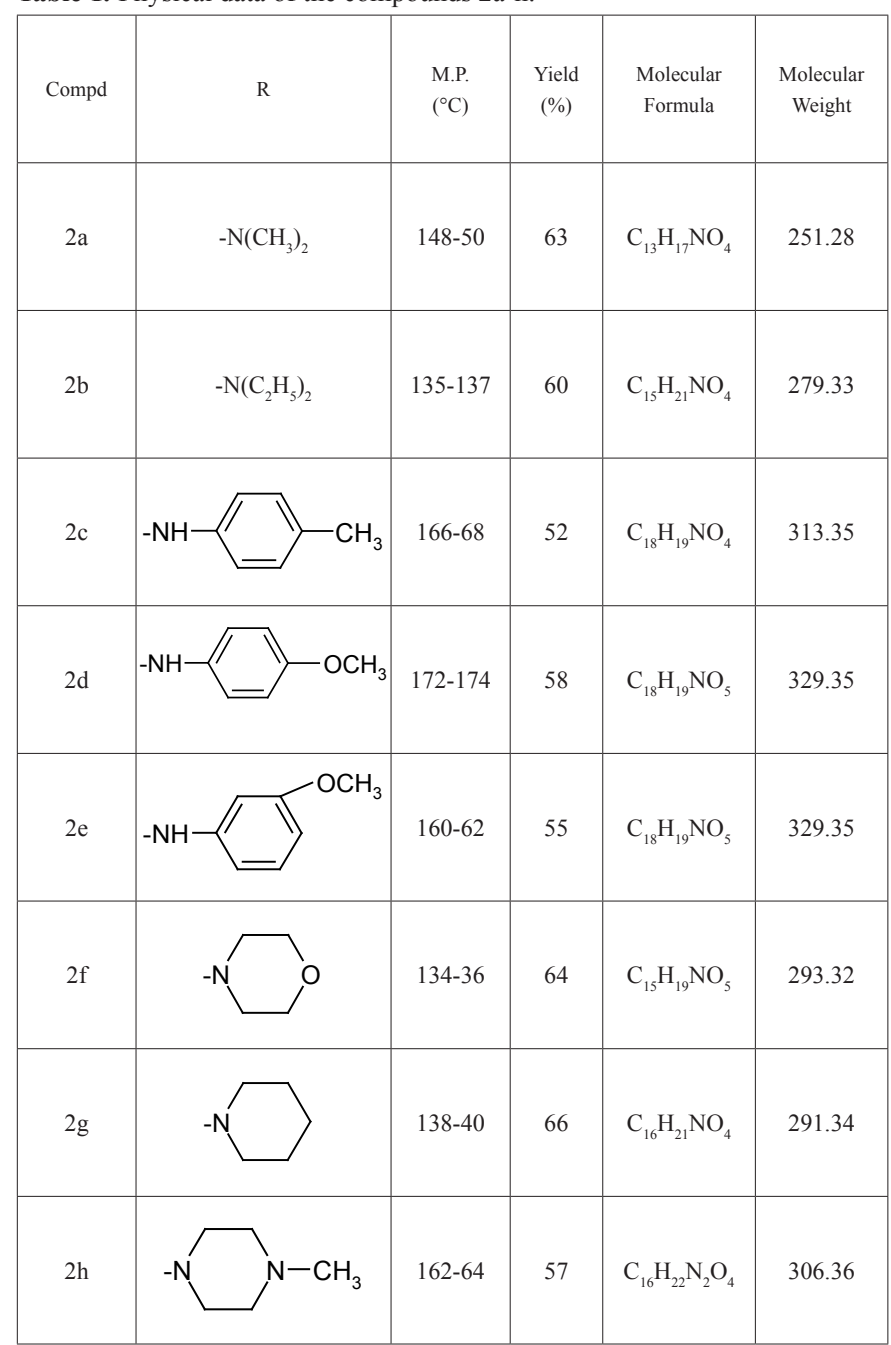

Antibacterial and antifungal activity

All the synthesized compounds were screened for in vitro antibacterial and antifungal activities at the concentration of 100 and $200 \mu \mathrm{g} / \mathrm{mL}$. The bacterial 
strains gram positive (Bacillus subtilis ATCC 6633 \& Staphylococcus aureus ATCC 25923), gram negative (Escherichia coli ATCC 8739) and fungal strains (Candida albicans NCIM 300 \& Aspergillus flaveous NCIM 524) were used. Ofloxacin and voriconazole were used as reference drugs for comparison. Among the tested compounds, 1,1'-\{5-[(4-methylpiperazin-1-yl)methyl]-46dihydroxy-1,3-phenylene $\}$ diethanone $(\mathbf{2 h})$ showed very good activity against S. aureus, C. albicans and A. flaveous, similar type of activity exhibited by $1,1^{\prime}$ - $\{5$-[(4-methoxylphenylamino)methyl]-46-dihydroxy-1,3-phenylene $\}$ diethanone (2d) against $S$. aureus and A. flaveous. Both the compounds, $2 \mathbf{h}$ and 2d, also showed good activity against $E$. coli and B. subtilis. Rests of the compounds were moderate to low in their antimicrobial action (Table-II).
An analysis of results showed that the Mannich bases derived from methylpiperazine and $p$-anisidine $(\mathbf{2 h} \& \mathbf{2 d})$ were excellent in their antimicrobial action against the tested organisms. Highest activity was observed in the methylpiperazine derivative (2h), while replacing methylpiperazine with $p$-anisidine (2d) slightly decreased the activity and it was further observed that replacing it with $m$-anisidine (2e), morpholine (2f), piperidine $(\mathbf{2 g})$ and $p$-toludene (2c) the activity decreased significantly. Among the synthesized Mannich bases, the bases derived from dimethylamine (2a) and diethylamine (2b) showed lowest antibacterial activity. However, the activities of tested compounds are less than that of the standard agents used.

Table-II: Antibacterial and antifungal activities of the compounds 2a-h.

\begin{tabular}{|c|c|c|c|c|c|c|}
\hline \multirow{2}{*}{ Compounds } & \multirow{2}{*}{$\begin{array}{l}\text { Conc. }(\mu \mathrm{g} \backslash \\
\mathrm{mL})\end{array}$} & \multirow[b]{2}{*}{ E. coli } & \multicolumn{3}{|c|}{ Diameter of zone of inhibition (mm) } & \multirow[b]{2}{*}{ A. flaveous } \\
\hline & & & S. aureus & B. subtilis & C. albicans & \\
\hline Voriconazole & 20 & nt & $\mathrm{nt}$ & $\mathrm{nt}$ & 27 & 31 \\
\hline Ofloxacin & 20 & 27 & 30 & 29 & $\mathrm{nt}$ & $\mathrm{nt}$ \\
\hline $2 \mathrm{a}$ & $\begin{array}{l}100 \\
200\end{array}$ & $\begin{array}{l}11 \\
16\end{array}$ & $\begin{array}{l}13 \\
18\end{array}$ & $\begin{array}{l}10 \\
13\end{array}$ & $\begin{array}{l}12 \\
16\end{array}$ & $\begin{array}{c}9 \\
12\end{array}$ \\
\hline $2 b$ & $\begin{array}{l}100 \\
200\end{array}$ & $\begin{array}{l}10 \\
13\end{array}$ & $\begin{array}{l}12 \\
16\end{array}$ & $\begin{array}{c}9 \\
11\end{array}$ & $\begin{array}{c}8 \\
12\end{array}$ & $\begin{array}{c}6 \\
10\end{array}$ \\
\hline \multirow{2}{*}{$2 \mathrm{c}$} & 100 & 10 & 12 & 11 & 12 & 10 \\
\hline & 200 & 16 & 15 & 14 & 16 & 13 \\
\hline \multirow{2}{*}{$2 d$} & 100 & 17 & 20 & 18 & 19 & 18 \\
\hline & 200 & 22 & 24 & 21 & 25 & 23 \\
\hline \multirow{2}{*}{$2 \mathrm{e}$} & 100 & 15 & 18 & 14 & 16 & 15 \\
\hline & 200 & 20 & 21 & 19 & 22 & 18 \\
\hline \multirow[t]{2}{*}{$2 f$} & 100 & 12 & 15 & 13 & 12 & 14 \\
\hline & 200 & 18 & 19 & 17 & 18 & 20 \\
\hline \multirow{2}{*}{$2 \mathrm{~g}$} & 100 & 14 & 15 & 12 & 16 & 11 \\
\hline & 200 & 17 & 19 & 15 & 21 & 16 \\
\hline \multirow{2}{*}{$2 \mathrm{~h}$} & 100 & 18 & 23 & 15 & 20 & 21 \\
\hline & 200 & 21 & 26 & 22 & 27 & 25 \\
\hline
\end{tabular}

$\mathrm{nt}=$ not tested

\section{CONCLUSIONS}

As concluding remarks we obtained herein eight new Mannich bases from 4,6-diacetyl resorcinol. Findings revealed that Mannich reaction did not take place at the acetyl function but occurred at the 2-position into the aromatic ring. Among the synthesized compounds, two compounds (2h and $\mathbf{2 d}$ ) showed significant antibacterial and antifungal activity against the tested microbes.

\section{REFERENCES}

1.- H. I. Gul, A. A. Denizci, E. Erciyas, Arzneim-Forsch 52, 773, (2002).

2.- M. Ashok, B. S. Holla, B. Poojary, Eur. J. Med. Chem. 42, 1095, (2007).

3.- J. R. Dimmock, N. M. Kandepu, U. Das, G. A. Zello, K. H. Nienaber, Pharmazie 59, 502, (2004).

4.- S. N. Pandeya, D. Sriram, G. Nath, E. De Clercq, Eur. J. Med. Chem.35, 249, (2000).

5.- J. L. Wiley, I. D. Beletskaya, E. W. Ng, Z. Dai, P. J. Crocker, A. Mahadevan, R. K. Razdan, B. R. Martin, J. Pharmacol. Exp. Ther. 301, 679, (2002).

6.- M. S. Y. Khan, S. Sharma, A. Husain, Scientia Pharmaceutica 70, 287, (2002).

7.- R. Cruickshank, J. P. Dugid, D. P. Marmion, R. H. A. Swain, Medical Microbiology, vol 2, Churchill-Livingstone, Edinburgh, London, 1975. 